\title{
Brain edema after intracerebral hemorrhage in rats: The role of inflammation
}

\author{
Xiangjian Zhang ${ }^{\dagger}$, Haiyan $\mathrm{Li}^{\star}$, Shuchao Hü, Liying Zhang ${ }^{\star *}$, Chunyan Liu**, Chunhua Zhu', \\ Ruichun Liư, Chunyan $\mathrm{Li}^{\dagger}$
}

${ }^{\dagger}$ Department of Neurology, The Second Hospital of Hebei Medical University, Shijiazhuang, Hebei, 050000, *Department of Neurology, The Beijing Shijitan Hospital, ${ }^{* *}$ The Beijing Shijinshan Hospital, ${ }^{* *}$ Department of Neurology, The Second Hospital of Hebei Medical University, Shijiazhuang, Hebei, 050000, China

\begin{abstract}
Background: Intracerebral hemorrhage $(\mathrm{ICH})$ results in secondary brain edema and injury that may lead to death and disability. ICH also causes inflammation. It is unclear whether inflammation contributes to brain edema and neuron injury or functions in repairing the brain tissue. Aims: To understand the effect of inflammation in $\mathrm{ICH}$, we have carried out an investigation on the various aspects and the dynamic changes of inflammation. Settings and Design: An $\mathrm{ICH}$ model was generated by injecting $50 \mu \mathrm{l}$ autologous tail artery blood stereotactically into the right caudate nucleus of 30 rats, which were randomly divided into five $\mathrm{ICH}$ groups. Similarly, five Sham control groups were generated by inserting the needle to the right caudate nucleus of rats. Materials and Methods: Rat behavior was evaluated over the time course ( 6 h, $24 \mathrm{~h}, 48 \mathrm{~h}, 72 \mathrm{~h}$ and $7 \mathrm{~d}$ ) in each group. The rats were then killed by administering an overdose of pentobarbital. Following the euthanasia, the brain water content, neuronal loss, glia proliferation, inflammatory infiltration and brain morphology of the rats were measured. Additionally, the expression of TNF- $\alpha$, IL-6, ICAM-1, VEGF, $\mathrm{NF}-\mathrm{KB}, \quad \mathrm{C} 3$ and $\mathrm{CR} 2$ was analyzed by immunohistochemistry. Statistical Analysis: The data were analyzed by student's $t$ test. Results: Rat brain water content increased progressively over the time course and reached its peak at $48 \mathrm{~h}$ followed $\mathrm{ICH}$. The maximum of inflammatory infiltrate (especially neutrophils) and immunopositive cells of TNF- $\alpha$, IL-6 and NF- $\kappa B$, were at $48 \mathrm{~h}$. The expression of $\mathrm{C} 3$ and $\mathrm{CR} 2$ reached their peaks at $48-72 \mathrm{~h}$, while the expression ICAM-1 and VEGF were at maximum at $72 \mathrm{~h}$ followed ICH. Conclusions: The results suggested that the inflammatory cytokines, complement system and VEGF may have a function in the development of the brain edema and neuron injury followed $\mathrm{ICH}$.
\end{abstract}

Key words: Animal models, brain edema, inflammation, intracerebral hemorrhage

This article was supported by Hebei provicine, No.: 05276101D-27.
Intracerebral hemorrhage (ICH) is a common and one of the most devastating types of stroke. ICH accounts for $10-15 \%$ of all strokes, but it is responsible for approximately $50 \%$ of strokerelated deaths. It imparts some form of disability to $88 \%$ of its survivors. ${ }^{[1,2]}$ Secondary brain edema and brain damage may result in further deterioration in neurological function after ICH. Although the mechanisms of brain injury after ICH are not fully understood, several mechanisms appear to contribute to edema development. Inflammatory processes appear to be involved in cardiovascular and cerebrovascular disease. Previous reports showed that ICH was accompanied by inflammation. Whether the inflammation contributes to brain edema and neurological injury or plays a role in repairing the brain tissue is not known. In order to understand the role of inflammation after ICH and provide information for developing new therapeutic strategies, we carried out an investigation on the dynamic changes of inflammatory responses such as cytokines, complement and bloodbrain barrier breakdown.

\section{Materials and Methods}

\section{ICH rat model}

The Institutional Animal Care and use committee and the local experimental ethics committee have approved all experimental procedures. Sixty male Sprague-Dawley rats (13-15 weeks) were used. The rats were anesthetized with pentobarbital (40 mg/kg, IP) and positioned in a stereotaxic frame (SR-6N, Japan). A cranial burr hole $(1 \mathrm{~mm})$ was drilled on the right coronal suture $3.5 \mathrm{~mm}$ lateral to the midline. ICH was initiated in 30 rats stereotactically by infusing $50 \mu \mathrm{l}$ autologous tail artery blood into the right caudate nucleus at $10 \mu \mathrm{l} / \mathrm{min}$ through a 26 -gauge needle (coordinates: $0.2 \mathrm{~mm}$ anterior, $5.5 \mathrm{~mm}$ ventral and $3.5 \mathrm{~mm}$ lateral to bregma). Thirty rats served as sham controls with only a needle insertion. At the end the needle was removed, the burr hole was sealed with bone wax, the wound was sutured and the animal was 
placed in a warm box with free access to food and water. Physiological parameters were maintained in the normal range in this experiment.

\section{Behavior tests}

Four motor behavior tests were carried out on rats by observers blinded to the experimental group or Sham group at time points 6h, 24h, 48h, 72h and seven days postoperation. (1) Longa behavioral test, ${ }^{[3]}$ measured the spontaneous contralateral circling and tumble and was graded from 0 (no circling) to 4 (unconsciousness); (2) Berderson behavioral test, ${ }^{[4]}$ measured the palsy of the contralateral limbs and was graded from 0 (no palsy) to 3 (circling of the contralateral); (3) Beam walking test, ${ }^{[5]}$ measured the ability to walk on an 80-cm-long, 2.5-cm-wide wood beam and was graded from 0 for a rat that readily traversed the beam to 5 for a rat that was unable to move or fell off the beam; (4) Footfault asymmetry test, ${ }^{[6]}$ measured the ability to walk in a net (the areole was $2.3 \times 2.3 \mathrm{~cm}$ ).

\section{Brain water content measurement}

At time points $6 \mathrm{~h}, 24 \mathrm{~h}, 48 \mathrm{~h}, 72 \mathrm{~h}$ and seven days, after the neurobehavioral tests were done, each rat was killed by a pentobarbital overdose. The brain was quickly removed and placed on a cooled surface. The frontal pole (approximately $3 \mathrm{~mm}$ thick), ${ }^{[3,7]}$ the cerebellum and brainstem were removed. The cerebrum was coronally divided into three pieces by sectioning through the needle entry site and the midpoint of the posterior remnant, respectively. The first piece ( $2 \mathrm{~mm}$ thick) was cut ipsilateral and contralateral of ICH, the two sections were used for brain edema measurement. Each section was wrapped in preweighed aluminum foil and weighed to obtain the wet weight (WW), then dried for $72 \mathrm{~h}$ in an oven at $110^{\circ} \mathrm{C}$ and weighed again to obtain the dry weight (DW). Brain water content was calculated as the percentage change using the following formula: (WW- DW)/WW x $100 \%$.

\section{Histological examination}

The other two coronal specimens from the cerebrum were used for histological examination. Briefly, they were perfused with $4 \%$ paraformaldehyde in $0.1 \mathrm{~mol} / \mathrm{L}$ PBS (pH 7.4) for three days and then dehydrated and embedded in paraffin. Sections $(5 \mu \mathrm{m})$ were stained with hematoxylin and eosin or stained by routine immunohistochemical methods. Primary antibodies used were TNF- $\alpha$ (diluted 1:100, Santa Cruz, USA), IL-6 (diluted 1:50, Boshide company, China), ICAM-1 (diluted 1:100, Zhongshan biology technology company, China), VEGF (diluted 1:150, Zhongshan biology technology company, China), NF-кB (diluted 1:200, Santa Cruz, USA), C3 (diluted 1:500, Sigma, USA) and CR2 (complement receptor type 2, CD21) (diluted 1:500, Santa Cruz, USA). The secondary antibodies, secondary biotinylated conjugates and diaminobenzidine were from the Vector ABC kit (Zhongshan biology technology company, China). The immunohistochemical labeling and detection were performed following the protocols recommended by the manufacturers.

\section{Statistical analysis}

All the values were shown as mean and standard deviation (means $\pm \mathrm{SD})$, statistical analysis software SPSS 11.0 was used for data analysis. ANOVA and $q$ tests were carried out for water content data and student's $t$-test was carried out for the rest of the data. Differences were considered significant at $P<0.05$.

\section{Results}

\section{High neurological deficit scores at $24 \mathrm{~h}, 48 \mathrm{~h}$ and $72 \mathrm{~h}$ after operation}

The behavior tests showed that the maximum neurological deficit scores in both ICH and Sham groups were at $6 \mathrm{~h}$ postoperation, a phenomenon caused by the anesthesia and injuries during the operation [Figures 1.1-1.4]. The neurological deficit score of the ICH group maintained a high level at $24 \mathrm{~h}, 48 \mathrm{~h}$ and $72 \mathrm{~h}$, but decreased to the lowest level at seven days when the rats had recovered from the operation. In contrast, the scores of Sham control rats were lower than that of the ICH rats at $24 \mathrm{~h}, 48 \mathrm{~h}$ and $72 \mathrm{~h}$. The differences of the four behavior tests between the two groups were statistically significant, the $P$ values at $24 \mathrm{~h}, 48 \mathrm{~h}$, $72 \mathrm{~h}$ were $0.011,0.008,0.002$ respectively for the Longa test [Figure 1.1], 0.003, 0.012 and 0.001 for the Berderson test [Figure 1.2], 0.02, 0.02 and 0.002 for the Beam Walking test [Figure 1.3], and 0.041, 0.021, 0.004 for the Footfault asymmetry test [Figure 1.4].

\section{Significant difference of brain water content between $\mathrm{ICH}$ and Sham rats at $48 \mathrm{~h}, 72 \mathrm{~h}$}

Brain water content of the ICH and Sham groups showed no significant difference at $6 \mathrm{~h}(P=0.157)$ and $24 \mathrm{~h}(P=0.830)$ [Figure 2]. At 48h and 72h, the brain water content of both ICH ipsilateral and contralateral samples was significantly higher than those of Sham group $(P<0.001)$, with maximum water content observed at 48h. Significant differences were also observed between the ipsilateral and contralateral samples at $48 \mathrm{~h}(P=0.0430)$ and $72 \mathrm{~h}(P=0.049)$.

\section{Significant changes in the brain tissue of rats in $\mathrm{ICH}$ group}

A spherical hematoma was observed in the caudate nucleus area at all time points after ICH. At time point $6 \mathrm{~h}$, a few rounded scattered neutrophils were found around the periphery of the hematoma. No morphological changes of neurons were observed. At $24 \mathrm{~h}$, brain edema around the hematoma was visible, the infiltrated inflammatory cells were mainly mononuclear and several neutrophils could be seen. At 48h, the brain edema around the hematoma was pronounced and the brain tissue was diffluent and necrotic [Figure 3]. The hematoma was surrounded by a compact band of cells including viable neutrophils, some cell debris, a few macrophages and rare clusters of intact erythrocytes. Degenerated neurons with vague nucleus and disappearing Nissl bodies and neuronophagia were also observed. At 72h, hematoma 


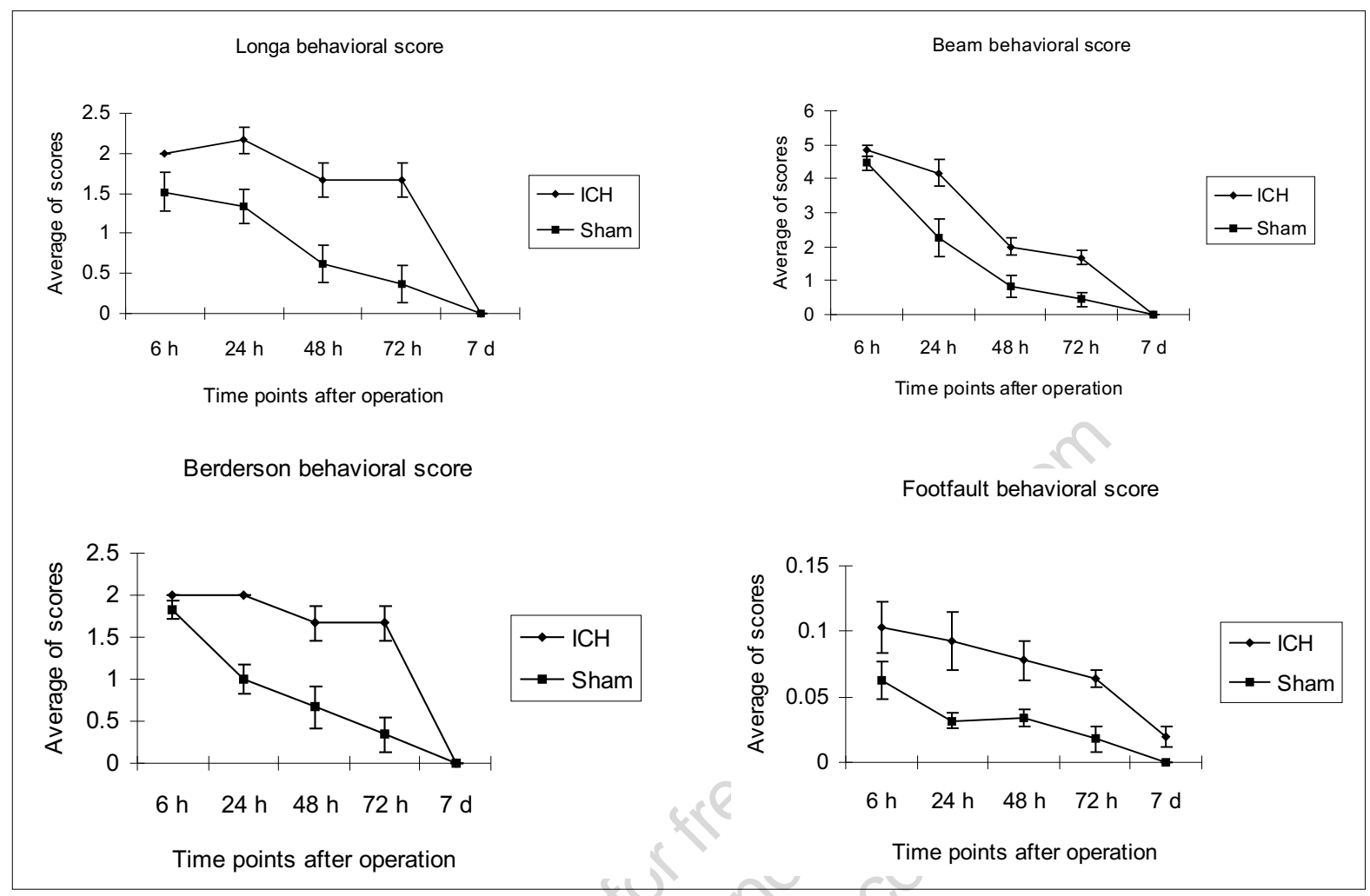

Figure 1: 1.1: Longa test, 1.2: Berderson test, 1.3: Beam walking test, 1.4: Footfault asymmetry test

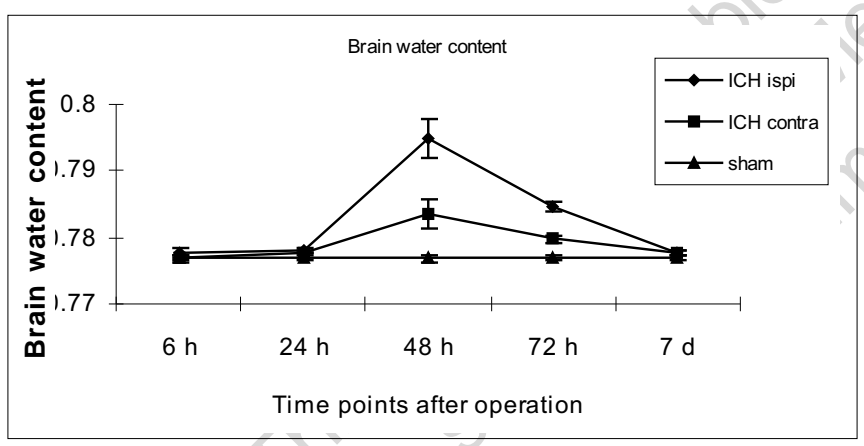

Figure 2: Dynamic tendency of brain water content over the time course. The lines represent ipsilateral, contralateral brain tissue after $\mathrm{ICH}$ and Sham group respectively

was surrounded by scattered glia cell containing hemosiderin, hyperplasia of glia and neovascularization. At time point seven days, the hematoma was resolving. The glia cell hyperplasia and neovascularization were abundant. Neutrophils were not present. In contrast, no hematoma or significant changes were observed in the brain tissue of rats from Sham group.

\section{Expression of inflammatory factors}

Positively stained neurons with a nucleus and normal morphology were counted as activated cells in three nonoverlapping regions under the microscope for the ipsilateral perihemotoma brain tissues. The expression of TNF- $\alpha$ [Figure 4], ICAM-1 [Figure 5], IL-6 [Figure 6] and NF-кB [Figure 7] after ICH were mainly in the cytoplasm of neurons and glia and reached their maximum at $48 \mathrm{~h}$ or $72 \mathrm{~h}$. The expression of

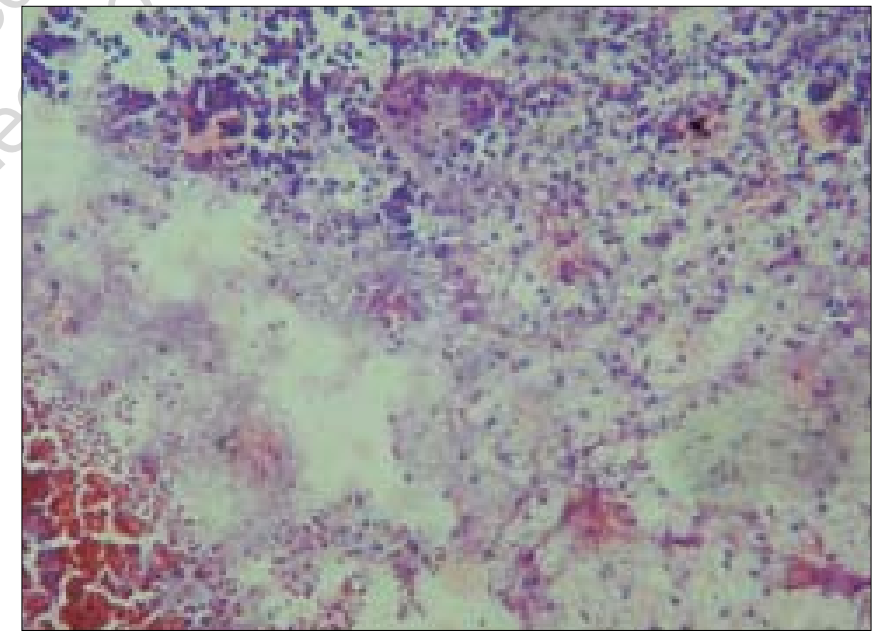

Figure 3: The brain edema around hematoma was obvious at $48 \mathrm{~h}$. They were diffluent, necrotic and surrounded by a compact band of cells including viable neutrophils, some cell debris, a few macrophages and rare clusters of intact erythrocytes. (H/E, 200x)

complement 3 [Figure 8] and CR2 [Figure 9] after ICH was up-regulated on the neurons, glia and endothelial cells in the perihemotoma. The expression of VEGF was up-regulated and maintained at high level after $48 \mathrm{~h}$, immunopositive cells were mainly neutrophils, neurons and endothelial cells [Figure 10].

\section{Discussion}

\section{Animal model}

Several experimental models of ICH have been described..$^{[8-10]}$ 


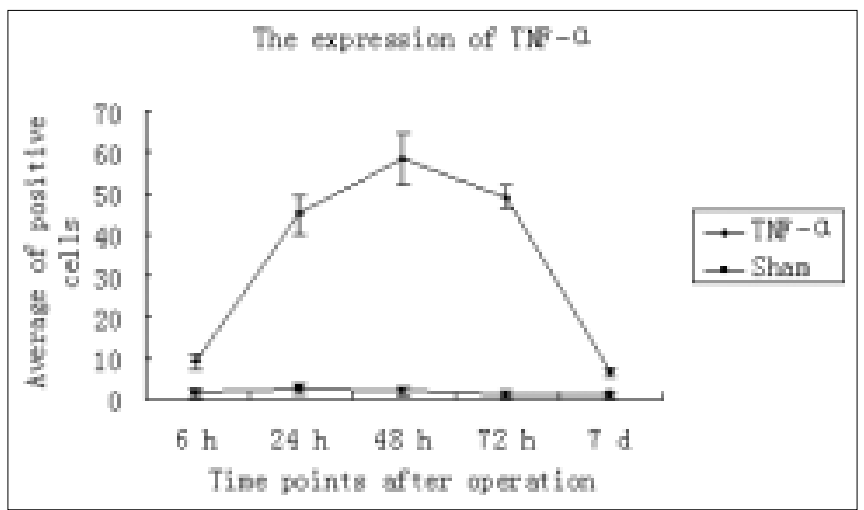

Figure 4: Dynamic expression tendency of TNF- $\alpha$ in the brain tissues around hematoma in rats from the $\mathrm{ICH}$ and Sham groups at different time points

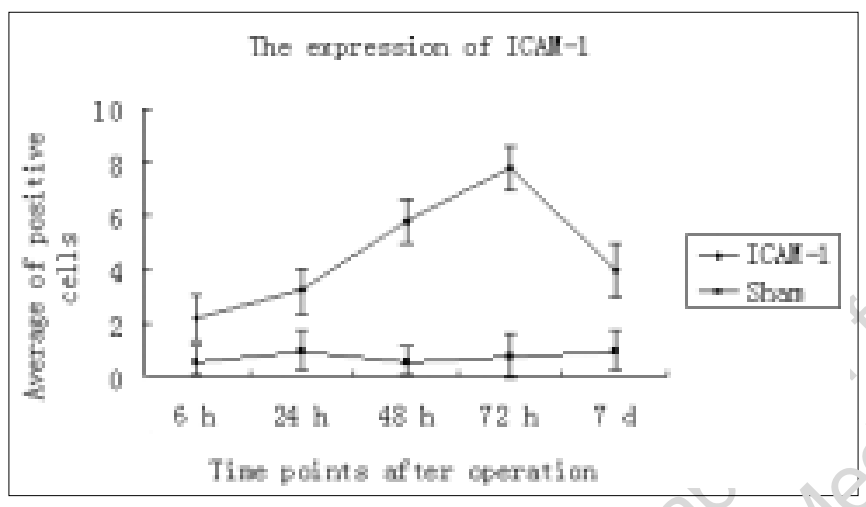

Figure 5: Dynamic expression tendency of ICAM-1 in the brain tissues around hematoma in rats from the $\mathrm{ICH}$ and Sham groups at different time points

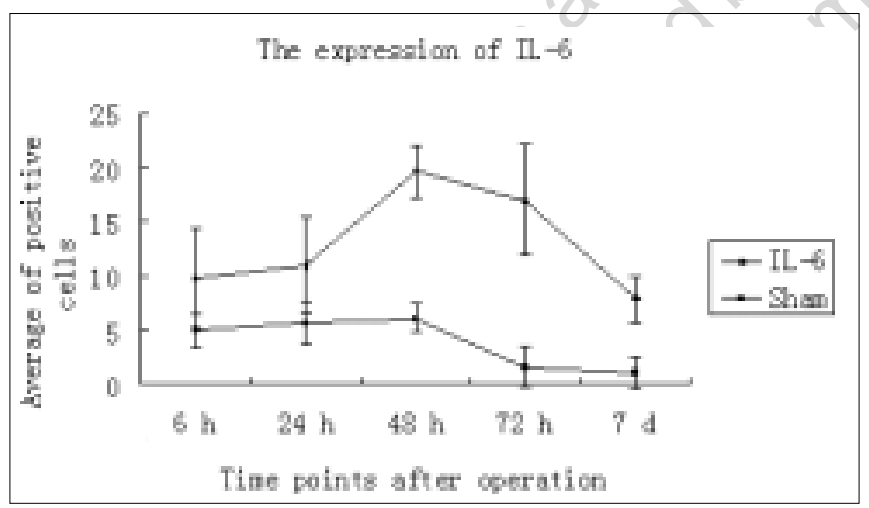

Figure 6: Dynamic expression tendency of IL-6 in the brain tissues around hematoma in rats from the $\mathrm{ICH}$ and Sham groups at different time points

With modification of previous ones, here we developed an ICH rat model by sampling the blood from the tail artery instead of the femoral artery. The modifications made the model more reliable since the procedure had no effect on the neurobehavioral evaluation.

\section{Brain water content and neurobehavioral evaluation}

We demonstrated that the brain edema reached its peak between $48 \mathrm{~h}$ and $72 \mathrm{~h}$ and resolved at seven days after ICH, consistent with that of the neurobehavioral deficit scores. This

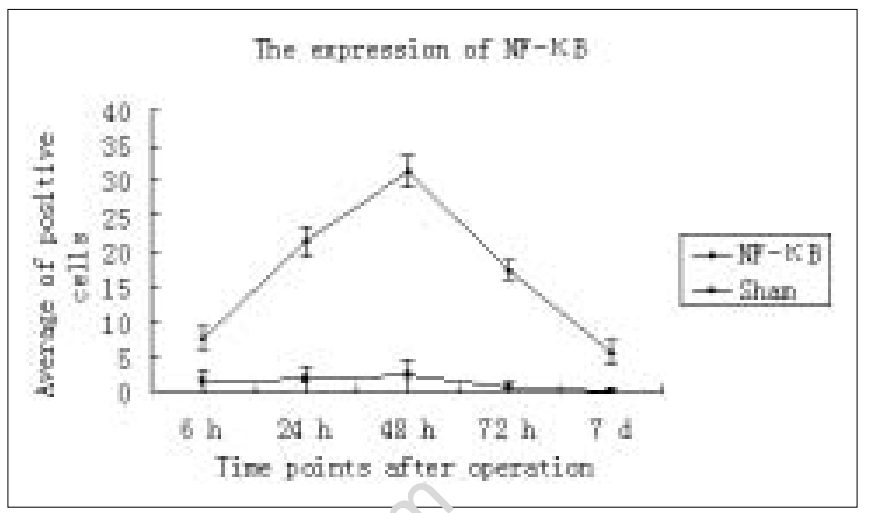

Figure 7: Dynamic expression tendency of NF- $\mathrm{KB}$ in the brain tissues around hematoma in rats from the $\mathrm{ICH}$ and Sham groups at different time points

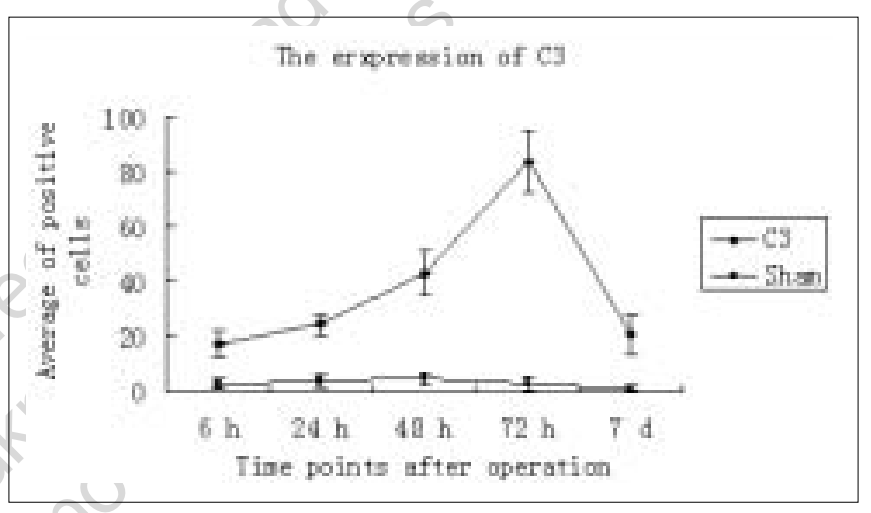

Figure 8: Dynamic expression tendency of complement 3 in the brain tissues around hematoma in rats from the $\mathrm{ICH}$ and Sham groups at different time points

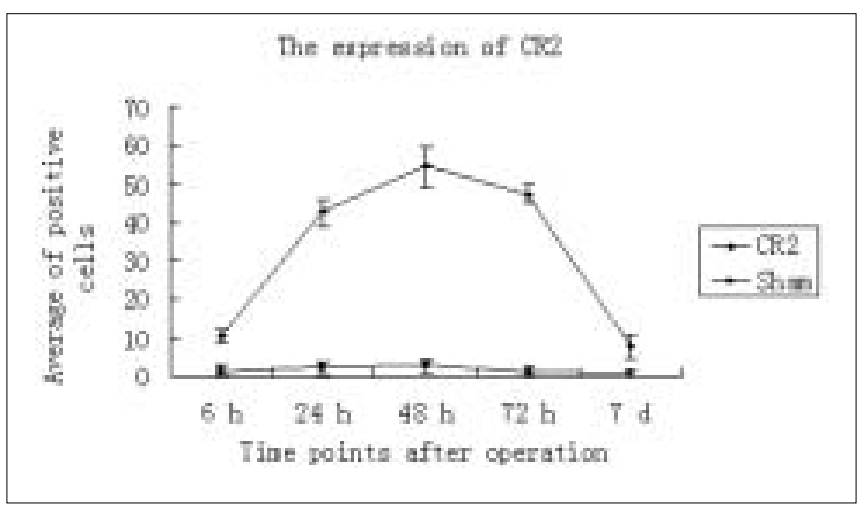

Figure 9: Dynamic expression tendency of CR2 in the brain tissues around hematoma in rats from the $\mathrm{ICH}$ and Sham groups at different time points

suggested that there was a possible correlation between brain edema and motor behavior changes. Since four neurobehavioral tests were used for evaluation of the motor behavior, the neurobehavioral deficit scores should be more reliable.

\section{Inflammatory cytokines: TNF- $\alpha$, IL-6, ICAM-1 and NF- $\kappa B$}

Neutrophilic inflammation is in the vicinity of cerebral hematoma. Neutrophils release a variety of cytokines which might 


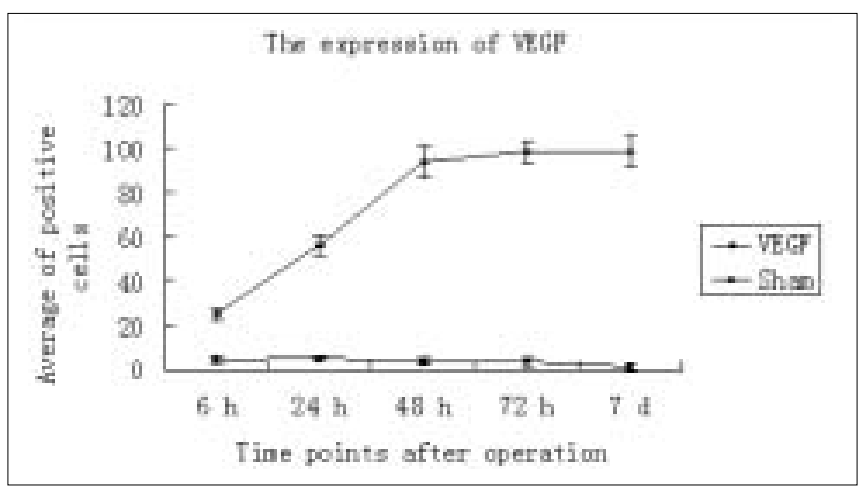

Figure 10: Dynamic expression tendency of VEGF in the brain tissues around hematoma in rats from the $\mathrm{ICH}$ and Sham groups at different time points

play an important role in brain edema formation and aggravation, such as TNF- $\alpha$, ICAM-1, IL-6 and NF- $\kappa$ B. ${ }^{[11-13]}$ It is also characterized by leukocyte behavior changes in the microvessels. Many leukocytes roll and adhere to the postcapillary venule and capillary walls and then these neutrophils infiltrate and migrate outside the vascular walls and move into the parenchyma. This inflammatory response is associated with expression of inflammatory mediators, including inflammatory cytokines, ${ }^{[14]}$ chemokines $^{[15]}$ and adhesion molecules. ${ }^{[16]}$ In turn, infiltrated neutrophils release proteases and oxidases and result in secondary brain injury. ${ }^{[17]}$ Our results indicated that neutrophils around the hematoma were the most abundant at $48 \mathrm{~h}$ to $72 \mathrm{~h}$ postoperation; the amount of inflammatory cytokines such as IL- 6 and TNF- $\alpha$ increased and reached their peaks at $48 \mathrm{~h}$ after ICH. The expression of ICAM-1 was up-regulated, correlated with the action of IL- 6 and TNF- $\alpha$ after ICH. Additionally the immunopositive ICAM-1 neurons were the most abundant at 72h. ICAM-1 neurons might contribute to the adhesion of activated microglias to neurons, leading to the neuron injury and brain edema after ICH. Transcription factor NF- $\mathrm{BB}$ plays a key role in secondary impairments of the tissues around the hematoma ${ }^{[18,19]}$ by inducing the expression of various genes related to cell injury and apoptosis. Here we demonstrated that the expression of $\mathrm{NF}-\kappa \mathrm{B}$ was consistent with the time course of brain edema after ICH, suggesting that NF-кB may worsen the brain edema and brain injury.

\section{Complement system: $\mathrm{C} 3$ and CR2}

C3 is an indicator of complement activation, ${ }^{[20]} \mathrm{C} 3 \mathrm{~d}$ is a degradation product of activated $\mathrm{C} 3$. $\mathrm{CR} 2$ is a receptor for $\mathrm{C} 3 \mathrm{~d}$. It belongs to a family of complement regulatory proteins and is known for its bridging function at the intersection of innate host defense and acquired humoral immunity. ${ }^{[21]}$ Possibly by the adjuvant effect of $\mathrm{C} 3 \mathrm{~d}$, the $\mathrm{C}$ system (C3 and $\mathrm{CR} 2$ ) may be involved in selecting antigens for recognition by the acquired immune system. This selecting antigen function is an immunityaugmenting function. The enhancement magnitude of antigen attachment of C3d might be 10,000-fold, ${ }^{[21,22]}$ which is far greater than that of complete Freund's adjuvant. It showed that local activation of complement in the brain is of pathophysiological significance in both degenerative and inflammatory neurological diseases including cerebrovascular disorders. Complement depletion significantly reduced edema formation at both 24 and $72 \mathrm{~h}$ after ICH. ${ }^{[23,24]}$ We demonstrated the up-regulation of C3 and $\mathrm{CR} 2$ protein levels in response to ICH in rat brain, suggesting C3 and CR2 may play a role in the development of inflammation, brain edema and brain injury after ICH.

\section{VEGF}

The results showed that the VEGF in rat brain after ICH was up-regulated. The up-regulation of VEGF correlates with bloodbrain barrier breakdown and vasogenic brain edema formation. VEGF expression reached its peak at 72h. However, many positive cells, small vessel and vessel-like structures were still observed at seven days, suggesting that VEGF promoted the late stage of vasogenic edema. VEGF accelerates the breakdown of endothelial cells and the basilar membrane, which could subsequently increase the leukocyte infiltration and aggravate brain edema, ${ }^{[25]}$ VEGF might stimulate the angiogenic response and neovascularization. ${ }^{[26]}$

\section{Conclusion}

This study characterized the brain edema, neurobehavioral deficit and inflammatory response after ICH in rat. Our results showed that cytokines, complement system and blood-brain barrier breakdown might play a role in the development of the brain edema and neuron injury after ICH. Our work will help to shed light on understanding of how the brain modulates inflammatory injury responses.

\section{Acknowledgment}

We would like to thank technician Hongran Wu and Bianfen Jin for their technical assistance.

\section{References}

1. Tayior CL, Selman WR, Ratcheson RA. Brain attack. The emergent management of hypertensive hemorrhage. Neurosurg Clin N Am 1997;8:237-44.

2. Sacco RL, Mayer SA. Epidemiology of intracerebral hemorrhage. In: Feldmann E, editor. Intracerebral Hemorrhage. Futura: Mount Kisco, NY; 1994. p. 3-23.

3. Longa EZ, Weinstein PR, Carlson S, Cummins R. Reversible middle cerebral artery occlusion without craniotomy in rats. Stroke 1989;20:84-91.

4. Berderson JB, Pitts LH, Tsuji M, Nishimura MC, Davis RL, Bartkowski H. Rat middle cerebral artery occlusion: Evaluation of the model and development of neurologic examination. Stroke 1986;17:472-6.

5. Altumbabic M, Peeling J, Bigio MR. Intracerebral hemorrhage in the rat: Effects of hematoma aspiration. Stroke 1998,29:1917-23.

6. Bland ST, Schallert T, Strong R, Aronowski J, Grotta JC, Feeney DM. Early exclusive use of the affected forelimb after moderate transient focal ischemia in rats: Function and anatomic outcome. Stroke 2000,31:1144-52.

7. Xi GH, Hua Y, Bhasin RR, Ennis SR, Keep RF, Hoff JT. Mechanism of edema formation after intracerebral hemorrhage: Effect of extravasated red blood cells on blood flow and blood-brain barrier integrity. Stroke 2001;32:2932-8.

8. Xi G, Hua Y, Keep RF, Younger JG, Hoff JT. Systemic complement depletion diminishes perihematomal brain edema. Stroke 2001;32:162-7.

9. Lee KR, Colon GP, Betz AL, Keep RF, Kim S, Hoff JT. Edema from intracerebral hemorrhage: The role of thrombin. J Neurosurg 1996;84:91-6.

10. Hua Y, Schallert T, Keep RF, Wu J, Hoff JT, Xi G. Behavioral tests after intracerebral hemorrhage in the rat. Stroke 2002;33:2478-84.

11. Arvin B, Neville LF, Barone FC, Feuerstein GZ. The role of inflammation and cytokines in brain injury. Neurosci Biobehav Rev 1996;20:445-52. 
12. Ghirnikar RS, Lee YL, Eng LF. Inflammation in traumatic brain injury: Role of cytokines and chemokines. Neurochem Res 1998;23:329-40.

13. Munoz-Fernandez MA, Fresno M. The role of tumour necrosis factor, interleukin 6 , interferon-gamma and inducible nitric oxide synthase in the development and pathology of the nervous system. Prog Neurobiol 1998;56:307-40.

14. Feuerstein GZ, Liu T, Barone FC. Cytokines, inflammation and brain injury: Role of tumor necrosis factor-alpha. Cerebrovasc Brain Metab Rev 1994;6:341-60.

15. Furie MB, Randolph G.J. Chemokines and tissue injury. Am J Pathol 1995;146:1287-301.

16. Yang GY, Gong C, Qin Z, Ye W, Mao Y, Betz AL. Inhibition of TNF- $\alpha$ attenuates infarct volume and ICAM-1 expression in ischemic mouse brain. Neuroreport 1998;9:2131-4

17. Del Bigio MR, Yan HJ, Buist R, Peeling J. Experimental intracerebral hemorrhage in rats: Magnetic resonance imaging and histopathological correlates. Stroke $1996 ; 27: 2312-20$.

18. Karibe H, Niizuma H, Ohyama H, Shirane R, Yoshimoto T. Hepatitis C virus (HCV) infection as a risk factor for spontaneous intracerebral hemorrhage: Hospital based case-control study. J Clin Neurosci 2001;8:423-5.

19. Hang CH, Shi JX, Li JS, Wu W, Yin HX. Concomitant upregulation of nuclear factor-kB activity, proinflammatory cytokines and ICAM-1 in the injured brain after cortical contusion trauma in a rat model. Neurol India 2005;53:312-7.

20. Bellander BM, von Holst H, Fredman P, Svensson M. Activation of the complement cascade and increase of clusterin in the brain following a cortical contusion in the adult rat. J Neurosurg 1996;85:468-75.
21. Dempsey PW, Allison ME, Akkaraju S, Goodnow CC, Fearon D'T. C3d of complement as a molecular adjuvant: Bridging innate and acquired immunity. Science $1996 ; 27: 348-50$

22. Zhang X.J, McGeer PL. Correlation of complement receptor type 2 with senile plaque and neurofrillar tangles in the hippocampal and temporal isocortex brain tissue in patients with Alzheimer disease. Zhongguo Linchuang Kangfu 2004;8:6212-4.

23. Hua Y, Xi G, Keep RF, Hoff JT. Complement activation in the brain after experimental intracerebral hemorrhage. J Neurosurg 2000;92:1016-22.

24. Xi G, Hua Y, Keep RF, Younger .JG, Hoff .JT. Brain edema after intracerebra hemorrhage: The effects of systemic complement depletion. Acta Neurochir Suppl $2002 ; 81: 253-6$.

25. Fischer S, Renz D, Schaper W, Karliczek GF. In vitro effects of dexamethasone on hypoxia-induced hyperpermeability and expression of vascular endothelial growth factor. Eur J Pharmacol 2001;411:231-43

26. Krum JM, Khaibullina A. Inhibition of endogenous VEGF impedes revascularization and astroglial proliferation: Roles for VEGF in brain repair. Exp Neurol 2003;181:241-57.

\section{Accepted on $07-08-2006$}

Source of Support: Nil, Conflict of Interest: None declared.

\section{Author Help: Sending a revised article}

1) Include the referees' remarks and point to point clarification to those remarks at the beginning in the revised article file itself. In addition, mark the changes as underlined or coloured text in the article. Please include in a single file

a. referees' comments

b. point to point clarifications on the comments

c. revised article with text highlighting the changes done

2) Include the original comments of the reviewers/editor with point to point reply at the beginning of the article in the 'Article File'. To ensure that the reviewer can assess the revised paper in timely fashion, please reply to the comments of the referees/editors in the following manner.

- $\quad$ There is no data on follow-up of these patients.

Authors' Reply: The follow up of patients have been included in the results section [Page 3, para 2]

- $\quad$ Authors should highlight the relation of complication to duration of diabetes.

Authors' Reply: The complications as seen in our study group has been included in the results section [Page 4, Table] 